Neutron Capture and Fission Measurements on Actinides at DANCE

A. Chyzh, C. Y. Wu, E. Kwan, R. Henderson, J. Gostic, J. Ullmann, J. O'Donnell, T. A. Bredeweg, M. Jandel, A. Couture, R. C. Haight, H. Y. Lee

October 13, 2011

CGS-14

Guelph, Canada

August 28, 2011 through September 2, 2011 
This document was prepared as an account of work sponsored by an agency of the United States government. Neither the United States government nor Lawrence Livermore National Security, LLC, nor any of their employees makes any warranty, expressed or implied, or assumes any legal liability or responsibility for the accuracy, completeness, or usefulness of any information, apparatus, product, or process disclosed, or represents that its use would not infringe privately owned rights. Reference herein to any specific commercial product, process, or service by trade name, trademark, manufacturer, or otherwise does not necessarily constitute or imply its endorsement, recommendation, or favoring by the United States government or Lawrence Livermore National Security, LLC. The views and opinions of authors expressed herein do not necessarily state or reflect those of the United States government or Lawrence Livermore National Security, LLC, and shall not be used for advertising or product endorsement purposes. 


\title{
Neutron Capture and Fission Measurements on Actinides at DANCE
}

\author{
A. Chyzh*, C. Y. Wu, E. Kwan, R. A. Henderson, J. M. Gostic \\ Lawrence Livermore National Laboratory, \\ Livermore, CA 94550, USA \\ *E-mail: chyzh1@llanl.gov \\ www.llnl.gov
}

J. L. Ullmann, T. A. Bredeweg, M. Jandel, A. Couture,

J. M. O'Donnell, R. C. Haight, and H. Y. Lee

Los Alamos National Laboratory,

Los Alamos, NM 87545, USA

www.lanl.gov

\begin{abstract}
The prompt $\gamma$-ray energy and multiplicity distributions in the spontaneous fission of ${ }^{252} \mathrm{Cf}$ have been measured using a highly granular $4 \pi \gamma$-ray calorimeter. Corrections were made for both energy and multiplicity distributions according to the detector response, which is simulated numerically using a model validated with the $\gamma$-ray calibration sources. A comparison of the total $\gamma$-ray energy distribution was made between the measurement and a simulation by random sampling of the corrected $\gamma$-ray energy and multiplicity distributions through the detector response. A reasonable agreement is achieved between the measurement and simulation, indicating weak correlations between $\gamma$-ray energy and multiplicity. Moreover, the increasing agreement with increasing multiplicity manifests the stochastic aspect of the prompt $\gamma$ decay in spontaneous fission. This calorimeter was designed for the study of neutron capture reactions and an example is given, where the ${ }^{238} \mathrm{Pu}(\mathrm{n}, \gamma)$ measurement was carried out in the laboratory environment for the first time.
\end{abstract}

Keywords: Neutron capture, spontaneous fission, DANCE, Cf-252, Pu-238, gamma-ray energy, multiplicity.

\section{Introduction}

Understanding the fission process is important for the design of nuclear reactor and the safety of its operation. The prompt energy released in fission is derived from three components; namely, fission fragments and their prompt neutrons as well as $\gamma$ decays. Significant amount of work has been 
carried out for the measurement and modeling of the mass and kinetic energy distributions of fission fragments ${ }^{1}$ as well as the energy distribution and the average multiplicity of emitted neutrons. ${ }^{2,3}$ However, there is only limited measurement and modeling for the $\gamma$-ray decay.

With the advance of modern highly granular $4 \pi \gamma$-ray detector arrays such as the Detector for Advanced Neutron Capture Experiments $(\mathrm{DANCE})^{4}$ at Los Alamos Neutron Science Center (LANSCE), the precision measurement of the prompt $\gamma$-ray multiplicity as well as energy distribution in fission becomes feasible. The unique features of DANCE such as the nearly $\gamma$-ray energy independence of the multiplicity response, make it possible, for the first time, to unfold the $\gamma$-ray multiplicity distribution in fission. This enables one to quantify the correlation between $\gamma$-ray energy and multiplicity and advance our understanding of the prompt $\gamma$-ray decay process in fission.

Such a study has been carried out for the spontaneous fission of ${ }^{252} \mathrm{Cf}$. The prompt $\gamma$ rays have been measured by the DANCE array in coincidence with the detection of fission fragments by a parallel-plate avalanche counter (PPAC). The prompt $\gamma$-ray energy and multiplicity distributions were derived in the off-line analysis and their unfoldings were performed using both Bayesian ${ }^{5}$ and Singular Value Decomposition (SVD) ${ }^{6}$ methods according to the detector response. The study of correlation between $\gamma$-ray energy and multiplicity was made by investigating the total $\gamma$-ray energy between the measurement and simulation by random sampling their unfolded distributions through the detector response. Details of this measurement and results are presented below.

\section{Experiment}

A ${ }^{252} \mathrm{Cf}$ source with a strength $\sim 0.15 \mu \mathrm{C} i$ was prepared by stippling the material on a $3 \mu \mathrm{m}$ thick titanium foil, which was then covered by the 1.4 $\mu \mathrm{m}$ thick aluminized mylar before being inserted into the PPAC, to serve as a cathode. Two anodes, made of the same thickness aluminized mylar, were placed on both sides of the cathode at a distance of $3 \mathrm{~mm}$. The PPAC was operated with the isobutane gas pressure $\sim 4.00$ torr stabilized by a feedback loop of constant gas flow. It has efficiency $\sim 82 \%$ for the detection of fission fragments with a bias of $375 \mathrm{~V}$ applied on anodes and provides a fast and clean fission trigger for the DANCE array to record the coincident $\gamma$ rays from fission fragments with a time window $\sim 30 \mathrm{~ns}$. Details of the design and fabrication for this PPAC is described in Ref. ${ }^{7}$

DANCE is a $4 \pi \gamma$-ray calorimeter and consists of $160 \mathrm{BaF}_{2}$ crystals 
with equal solid-angle coverage. It is designed to study the neutron-capture reactions on small quantities of radioactive and rare stable nuclei. The neutron-capture event is recognized by the measured total $\gamma$-ray energy and the summed photopeak is equivalent to the reaction $Q$ value. Besides its use as a calorimeter for the study of the neutron-capture reaction, DANCE can be used for the precision measurement of the $\gamma$-ray energy and multiplicity distributions as well as the total $\gamma$-ray distribution in fission as long as the measurement is accompanied by a charged-particle detector, such as the PPAC mentioned above, for the fission-fragment detection.

The experiment was fielded at LANSCE over a period of five days. A total of $\sim 3 \times 10^{6}$ fission events were selected for the current study by placing a time window $\sim 10$ ns on the coincident time spectrum between DANCE and PPAC, where a time resolution $\sim 2 \mathrm{~ns}$ is achieved. The pulse height and timing information for both DANCE and PPAC were derived from the recorded waveforms by the $500 \mathrm{MS} / \mathrm{s}, 300 \mathrm{MHz}$ bandwidth Acqiris digitizers. The coincident $\gamma$-ray energy and multiplicity distributions were determined from the DANCE array for those fission events. To avoid the summing effect on the $\gamma$-ray energy distribution, the spectrum, shown in Fig. 1, was obtained by requiring the $\gamma$ ray detected by the $\mathrm{BaF}_{2}$ crystal without any adjacent crystals being triggered. The sensitivity of DANCE for the $\gamma$-ray spectroscopy study is clearly demonstrated, where the strength of $\gamma$ ray falling monotonously as a function of energy over a range of five decades until tapering off $\sim 8 \mathrm{MeV}$ is observed.

The $\gamma$-ray energy and multiplicity response for the DANCE array has been studied extensively. ${ }^{8,9}$ A physical model of DANCE together with the surrounding material was created to study the response numerically and the validation was carried out by the comparison between the measurement and simulation using GEANT $4{ }^{10}$ with three $\gamma$-ray calibration sources, ${ }^{22} \mathrm{Na}$, ${ }^{60} \mathrm{Co}$, and ${ }^{88} \mathrm{Y}$. Many unique features of DANCE are identified, such as the detection efficiency, the peak-to-total ratio, and the multiplicity distribution are all nearly independent of $\gamma$-ray energy ranging from $150 \mathrm{keV}$ up to 10 $\mathrm{MeV}$. For instance, the detection efficiency varies between 84 to $88 \%$, the peak-to-total is nearly constant $\sim 55 \%$, and the average multiplicity differs no more than $6 \% .{ }^{11}$ Note that the values of $\gamma$-ray energy and multiplicity were derived by lumping together adjacent crystals if triggered. Introducing the physical model of PPAC into the simulation reduces the efficiency by $4 \%$ and $1 \%$ for the $\gamma$-ray energy of $200 \mathrm{keV}$ and $1 \mathrm{MeV}$, respectively.

The neutron capture measurement for ${ }^{238} \mathrm{Pu}$ was carried out in Nov Dec, 2010, using the DANCE array. The isotopic enriched target (99.35\%) 

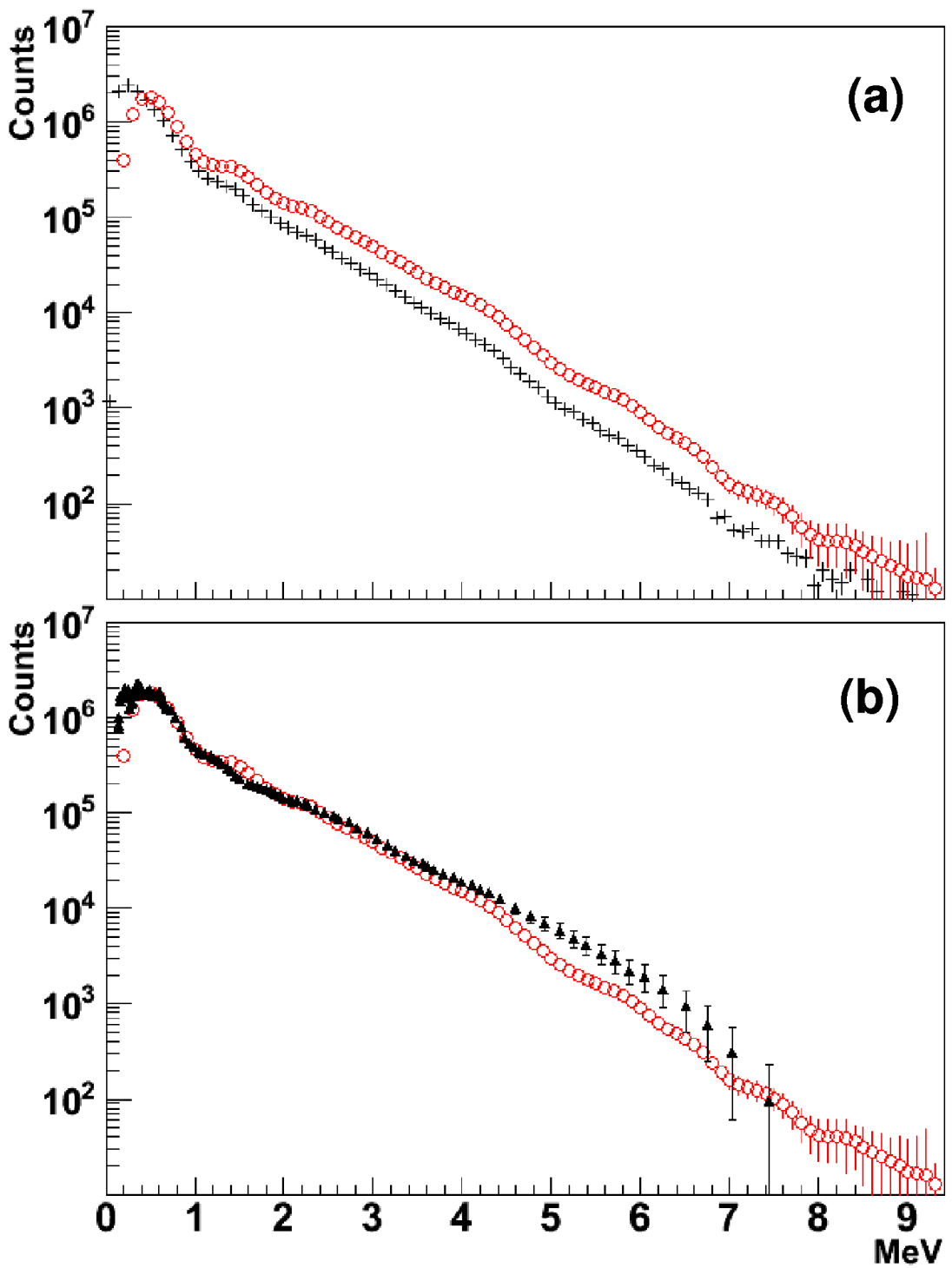

Fig. 1. Prompt $\gamma$-rays of the ${ }^{252} \mathrm{Cf}$ spontaneous fission. (a): measured (black crosses), unfolded (red circles). (b): Verbinski (black triangles), unfolded (red circles).

was prepared at LLNL with the new electroplating cell capable of depositing the isotope simultaneously on both sides of the $3-\mu \mathrm{m}$ thick Ti backing foil. A total mass of $395 \mu \mathrm{g}$ with an activity of $6.8 \mathrm{mCi}$ was deposited onto the 
area of $7 \mathrm{~mm}$ in diameter. The target was covered by $1.4 \mu \mathrm{m}$ double-side aluminized mylar before inserted into a vacuum-tight container. The total beam-on-target time was about 14 days.

\section{Results and discussion}

As was mentioned earlier, the least known quantity for the prompt energy released in the fission process is the $\gamma$ decay. The current work yields a precision measurement of the prompt $\gamma$-ray energy and multiplicity distributions for the spontaneous fission of ${ }^{252} \mathrm{Cf}$, which allows to quantify the correlations between $\gamma$-ray energy and multiplicity. However, before such a study can be carried out, the unfolding procedure is needed to correct those measured distributions according to the detector response.

The relationship between the measured distribution $\mathbf{M}_{\mathbf{o b s}}$ and the actual one $\mathbf{M}_{\mathbf{p h y s}}$ can be expressed as a matrix equation:

$$
\mathbf{M}_{\mathrm{obs}}=\mathbf{R} \cdot \mathbf{M}_{\mathbf{p h y s}}
$$

where $\mathbf{R}$ is the response matrix for a given detector system. In principle, one can derive the actual distribution from the measured one by inverting the response matrix, but in reality, this technique fails to produce sensible solutions in most cases. An extensive discussion on this subject and the techniques developed to achieve better solutions can be found in Ref. ${ }^{12}$ In our current work, the unfolding of measured $\gamma$-ray energy and multiplicity distributions was carried out using both the Bayesian and SVD methods implemented in the standalone software package. ${ }^{13}$ The results derived from both methods are essentially identical. More on the unfolding of measured energy and multiplicity by DANCE can be found in Ref. ${ }^{14}$

The response matrix of DANCE for both $\gamma$-ray energy and multiplicity was simulated numerically using GEANT4 according to the model validated by the $\gamma$-ray calibration sources discussed earlier. For the energy response, the simulation is restricted by the same experimental condition, which requires the $\gamma$-ray detected by the $\mathrm{BaF}_{2}$ crystal without any adjacent crystals being triggered. This reduces the average detection efficiency from $86 \%$ to $65 \%$ but the peak-to-total ratio remains the same $\sim 55 \%$. The unfolded $\gamma$-ray energy distribution together with the measured one is shown in Fig. 1(a) and the comparison with the previous measurement ${ }^{15}(\mathrm{~b})$ is shown in Fig. 1. The agreement is reasonable in general until $\sim 4 \mathrm{MeV}$ where the current data drops steeper toward higher $\gamma$-ray energy.

For the multiplicity response, the matrix was created by the simulations weighted by the unfolded $\gamma$-energy distribution to minimize the residual ef- 
fect of the nearly energy independence. The unfolded $\gamma$-ray multiplicity distribution together with the measured one is shown in Fig. 2(a) and the comparison with the semi-empirical formulated distribution ${ }^{16}$ is shown in Fig. 2(b). The agreement is good in general even though the current data shifts toward higher $\gamma$-ray multiplicity. Note that it is the first time that the actual $\gamma$-ray multiplicity in the spontaneous fission is derived experimentally.

To quantify the correlations between the $\gamma$-ray energy and multiplicity for the prompt $\gamma$ decay in spontaneous fission, a comparison of the total $\gamma$-ray energy was made between the measurement and a Monte Carlo simulation by random sampling of their unfolded distributions through the detector response of DANCE. The simulated total $\gamma$-ray energy distribution was obtained with one million samples selected according to the multiplicity distribution. A matching number of $\gamma$ rays with the chosen multiplicity was selected according the energy distribution before going through the detector response. Comparison between the measurement and simulation is shown in Fig. 3(a), where the agreement is reasonable, indicating weak correlations between the $\gamma$-ray energy and multiplicity.

More detailed study of their correlations was made by comparisons with the partition on the measured multiplicity, which shown in Fig. 3(b),(c),(d). The agreement in reproducing the average energy and variance is increasing with increasing multiplicity, which manifests the stochastic aspect of the prompt $\gamma$ decay in spontaneous fission of ${ }^{252} \mathrm{Cf}$. Obviously, the stochastic aspect can not account for all correlations without considering the details of nuclear structure of fission fragments. Nevertheless, this is an important finding to advance the modeling of the prompt $\gamma$ decay in fission.

The relative yield of neutron capture cross section for ${ }^{238} \mathrm{Pu}$ as a function of neutron incident energy is shown in Fig. 4. This spectrum was obtained by setting gates on the total energy $\mathrm{E}_{\text {tot }}=5.7-6.5 \mathrm{MeV}$ close to $5.646-\mathrm{MeV}$, the $\mathrm{Q}$-value of ${ }^{238} \mathrm{Pu}(n, \gamma)$, and the multiplicity, $\mathrm{M}=3-$ 4. This is to minimize the contribution from the non-negligible amount of daughter nucleus, ${ }^{234} \mathrm{U}$, and fission. Background subtraction was made for the residual contribution of ${ }^{234} \mathrm{U}$ and the contribution from neutrons elastically scattered off the container Kapton windows, the backing foil, and ${ }^{238} \mathrm{Pu}$ itself. A complementary experiment equipped with a fission counter will be fielded to measure the fission contribution quantitatively to complete the final analysis of the neutron capture cross section for ${ }^{238} \mathrm{Pu}$. 


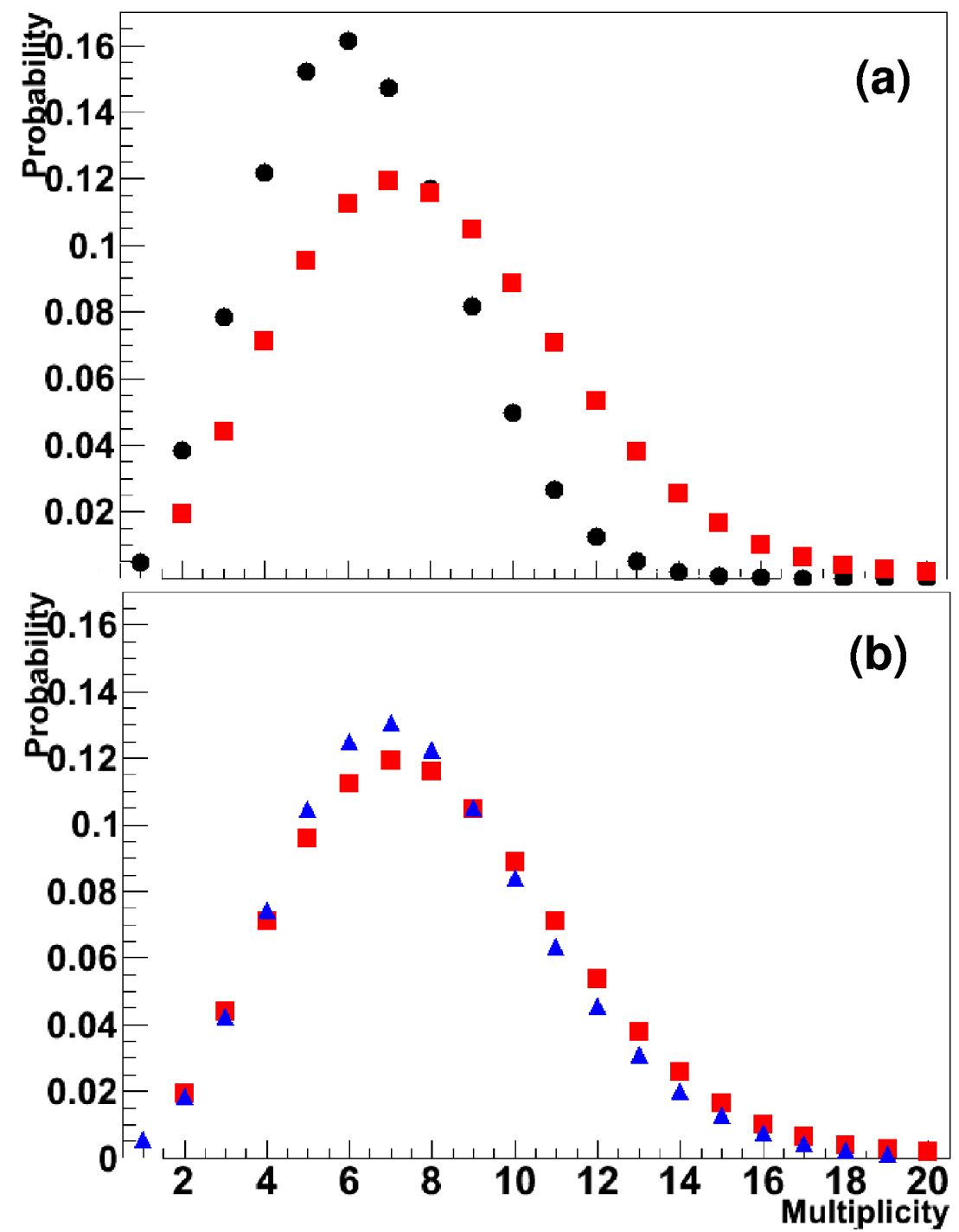

Fig. 2. Prompt $\gamma$-ray multiplicity of the ${ }^{252} \mathrm{Cf}$ spontaneous fission. (a): measured (black circles), unfolded (red squares). (b): Brunson (blue triangles), unfolded (red squares).

\section{Summary}

The prompt $\gamma$-ray energy and multiplicity distributions in spontaneous fission of ${ }^{252} \mathrm{Cf}$ were measured using the DANCE array. Both distributions 


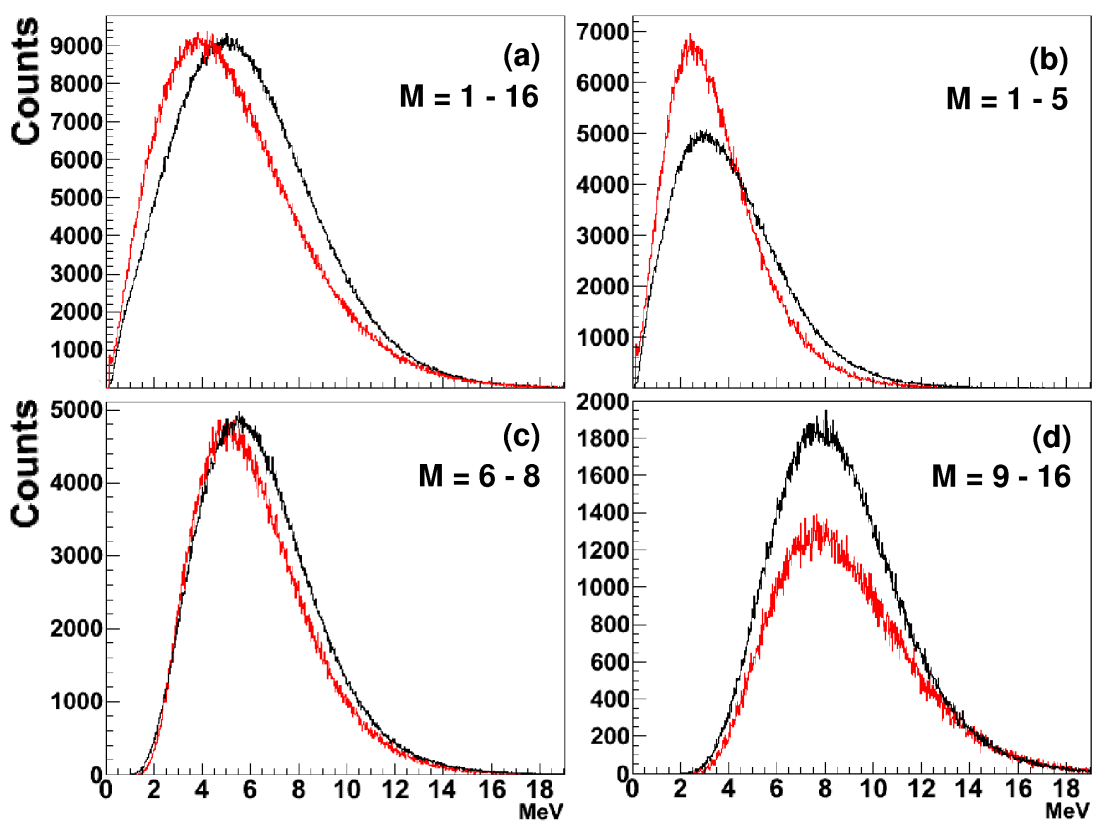

Fig. 3. Total $\gamma$-ray energy of the ${ }^{252} \mathrm{Cf}$ spontaneous fission: measured (black), simulated (red). (a): $M=1-16$. (b): $M=1-5$. (c): $M=6-8$. (d): $M=9-16$.

were unfolded using Bayesian and SVD methods and consistent results were obtained. A comparison of the total $\gamma$-ray energy distribution was made between the measurement and simulation by random sampling of both unfolded distributions through the detector response. A reasonable agreement between the measurement and simulation implies weak correlations between the $\gamma$-ray energy and multiplicity. The stochastic aspect is manifested by the increasing agreement with increasing multiplicity, which provides important physics for modeling the prompt $\gamma$ decay in the fission process. This measurement will be extended to study the correlations between the $\gamma$-ray energy and multiplicity for the prompt $\gamma$ decay in the neutron-induced fission.

An example is given for the neutron capture measurement using the DANCE array, where the relative cross section for the ${ }^{238} \mathrm{Pu}(\mathrm{n}, \gamma)$ reaction was derived. This reaction was measured for the first time in the laboratory environment. To complete the final determination of cross section, we need to subtract the fission contribution, which will be measured in an additional experiment equipped with a fission counter. 


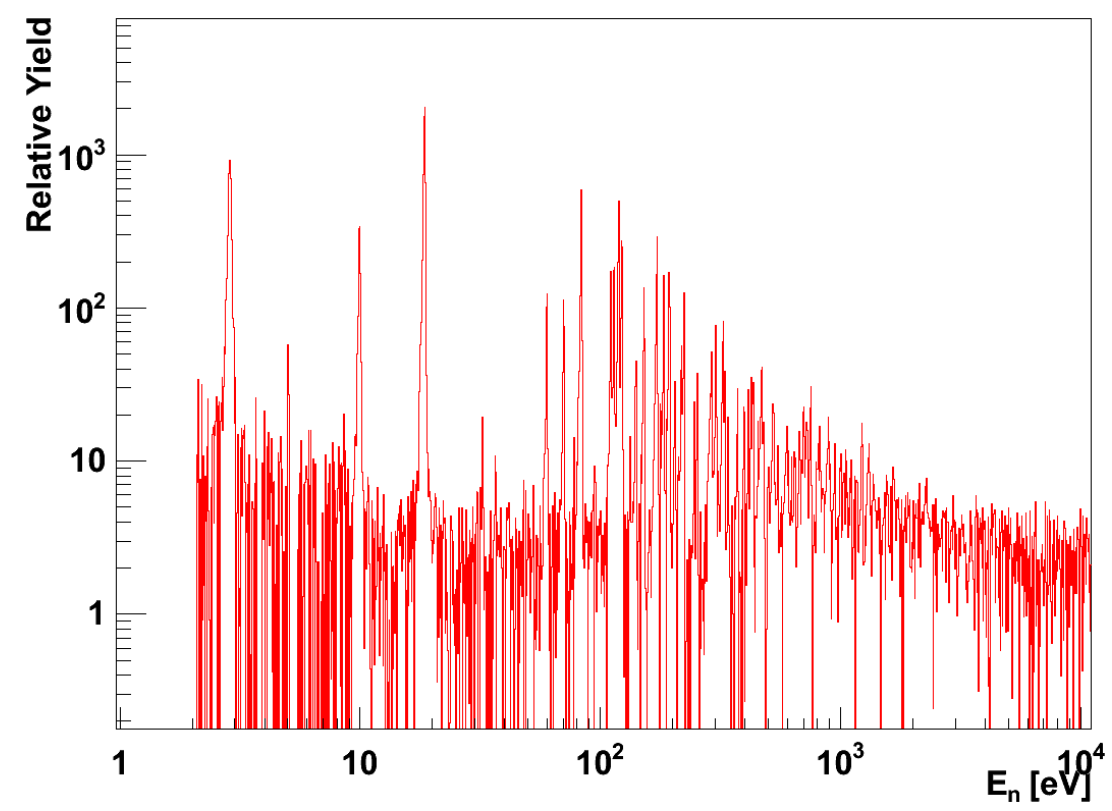

Fig. 4. The relative yield of the ${ }^{238} \mathrm{Pu}(n, \gamma)$ cross section.

We thank Prof. D. Gogny for many fruitful discussions during the course of this work. This work performed under the auspices of the US Department of Energy by Lawrence Livermore National Security, LLC under contract DEAC52-07NA27344 and by Los Alamos National Security, LLC under contract DE-AC52-06NA25396.

\section{References}

1. F. Gonnenwein, The Nuclear Fission Process, editor C. Wagemans, (CRC Press, 1991), p. 287 and references therein.

2. D.G. Madland and J.R. Nix, Nucl. Sci. Eng. 81, 213 (1982).

3. H.-H. Knitter, U. Brosa, and C. Budtz-Jorgensen, The Nuclear Fission Process, editor C. Wagemans, (CRC Press, 1991), p. 497 and references therein.

4. M. Heil et al., Nucl. Instrum. Methods in Phys. Res. A 459, 229 (2001).

5. G. D'Agostini, Nucl. Instrum. Mthods in Phys. Res. A 362, 487 (1995).

6. A. Höcker, V. Kartvelishvili, Nucl. Instrum. Methods in Phys. Res. A 362, 487 (1995).

7. C.Y. Wu et al., LLNL-TR-462118, Lawrence Livermore National Laboratory (2010).

8. R. Reifarth et al., LA-UR-03-5560, Los Alamos National Laboratory (2003). 
9. M. Jandel et al., Nucl. Instrum. Methods in Phys. Res. B 261, 1117 (2007).

10. S. Agostinelli et al., Nucl. Instrum. Methods in Phys. Res. A 506, 550 (2003).

11. A. Chyzh et. al., LLNL-TR-452298, Lawrence Livermore National Laboratory (2010).

12. G. Cowan, Statistical Data Analysis, (Clarendon Press, Oxford, 1998).

13. T.J. Adye, http://hepunx.rl.ac.uk/ adye/software/unfold/RooUnfold. html, RooUnfold 1.1.0.

14. A. Chyzh et al., LLNL-TR-460216, Lawrence Livermore National Laboratory (2010).

15. V.V. Verbinski, H. Weber, R.E. Sund, Phys. Rev. C 7, 1173 (1973).

16. G.S. Brunson Jr, LA-940B-T, Los Alamos National Laboratory (1982). 